\title{
3D Gait Analysis Based on UWB Wireless Body Area Networks
}

\author{
Fucheng $\mathrm{CaO}^{12}$ \\ ${ }^{1}$ College of Electronic Information and Engineering \\ Changchun University \\ Changchun, China \\ ${ }^{2}$ College of Communication Engineering \\ Jilin University \\ Changchun, China \\ e-mail: arthurcao@hotmail.com
}

\author{
Xin Li \\ College of Communication Engineering, \\ Jilin University \\ Changchun, China
}

Xilu Zhao

Saitama Institute of Technology

Saitama, 369-0293 Japan

\begin{abstract}
During the rehabilitation training, the methods of gait analysis and planning are very important. We must consider the simplicity of implementation and the validity of method used. Combined with ultra-wideband wireless body area network, gyroscopes, acceleration sensors and TOA positioning technology, we proposed a gait analysis method that is also effective in three-dimensional space. After summarizing the existing methods of gait analysis, we point out the shortcomings of commonly method based video motion capture and the advantages of ultra-wideband wireless sensor networks. Then, we propose a method for three dimensional gait analysis within UWB wireless body area networks. The acceleration and angular velocity data of each sensor unit were measured during le vel walking. Based on the positioning algorithm, the location, acceleration and angular velocity data of each node can be calculated during level walking by the UWB wireless sensor networks. Define global coordinate and local coordinate and combine, we are able to establish lower limb gait kinematic equations.
\end{abstract}

Keywords- Gait Analysis; Ultra-wideband; Wireless Body Area Networks, TOA, WSN

\section{INTRODUCTION}

The recent development of Wireless Body Area Networks (WBANs) arouses widespread concern. As one kind of small and low-power wearable wireless devices, it is on the verge of full new market needs in a variety of application such as distant posture detection, physiological or activity monitoring, wireless medical actuators and implants, assistance to medical diagnosis, motion capture for gaming or sports analysis, personal communications and multimedia etc. The architecture of WBANs is shown in Fig .l. Most of all, WBANs rely on emerging radio technologies such as Narrow-Band solutions at $2.4 \mathrm{GHz}$ based on e.g., Bluetooth - Low Energy, or Impulse Radio - Ultra Wideband (IR-UWB) solutions, which has the advantages of Ultra Low Power (ULP) consumption, low complexity, and low cost.

Federal Communications Commission (FCC) regulate that the frequency for the UWB technique is from 3.1 $\mathrm{GHz}$ to $10.6 \mathrm{GHz}$ in America. However, in Europe, the frequencies include two parts: from $3.4 \mathrm{GHz}$ to $4.8 \mathrm{GHz}$ and $6 \mathrm{GHz}$ to $8.5 \mathrm{GHz}$. The recent IEEE 802.15.6 standard dedicated to WBANs applications [11]. Nowadays, another rapid development of UWB technology in the wireless industry is the precise TOAbased indoor localization [1]. Some unique features of UWB make it very suitable for localization for WBANs in medical areas and it would not disturb the existing equipments because of the strict power radiation requirement.

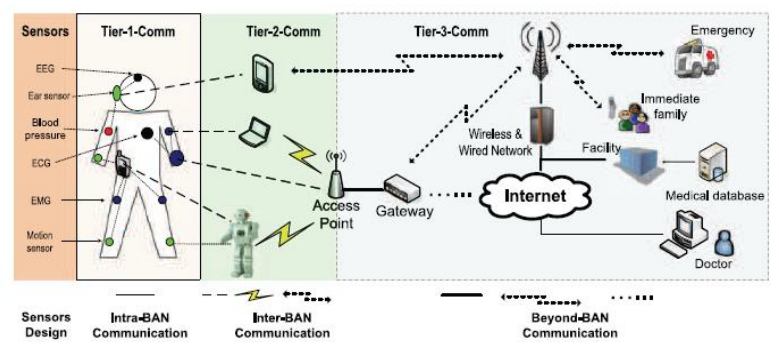

Figure 1. Architecture of body area network [3]

Considered the power consumption, WBAN nodes usually embed extremely low-power sensors and actuators, especially the wearable devices. In the near future, wearable systems could drastically change our daily life, by participating as local building components and key contributors into the Internet of Things. The architecture skeleton combined with WBANs could play a significant role in rehabilitation. The traditional motion capture with the video sensors at the body scale is shown in Fig .2 (from documentation of OpenSim).

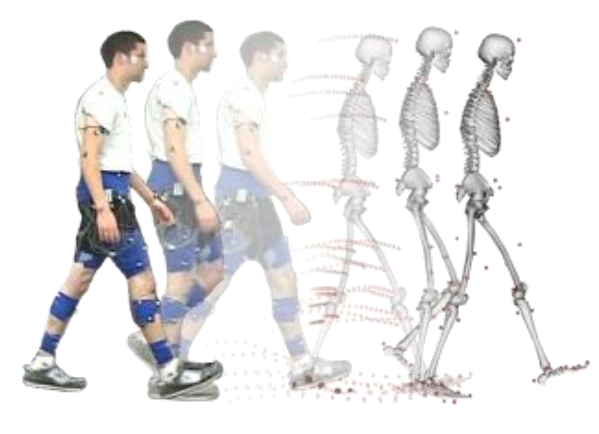




\section{REVIEW OF GAIT ANALYSIS TECHNOLOGIES}

There are a great many studies on the different aspects of the human gait. The following section contains a simple description of some studies on the newest technologies mainly used in human gait analysis and recognition. However, the studies of human gait mainly initially rely on the naked eye observation because of lacking of effective scientific and objective methods of measurement. Until recently, the application of instrumental gait analysis in clinical practice was quickly developed. With the development of computer technology and video cameras, many new method of gait analysis appeared. The method of optical systems is based on illuminated and reflective markers placed on the body. The localization of any on-body marker necessitates that the latter is viewed by at least two external cameras, which have known positions and orientations. In the image processing method, several digital or analog cameras with lens are used to gather gait-related information. Techniques such as threshold filtering are just some of the possible ways to gather data to measure the gait variables, by which the images are converted into black and white and calculate the number of light or dark pixels or background segmentation which simply removes the background of the image.

Fig .3 is an example of typical operating scenario and deployment of the F40 motion capture system at University of California. Such optical tracking systems are generally characterized by high localization accuracy and they are able to support real-time motion capture and/or navigation applications. However, they have limitations that may prevent from considering them in the very context, such as cost, complexity or the necessity to operate in geographically restricted and closed areas. On the other hand, vision motion capture system also suffer from non visibility problems, when the markers cannot be viewed by the surrounding cameras in cases of obstructions and/or obscurity conditions, and thus, the achieved accuracy can be reduced accordingly.

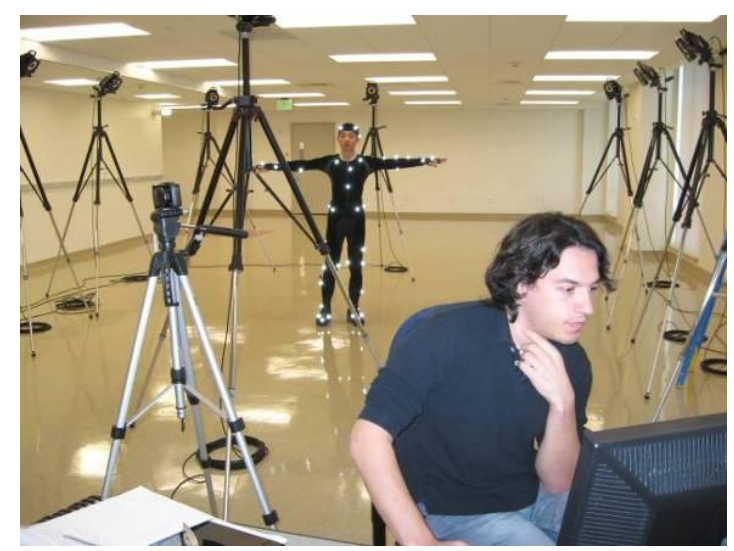

Figure 3. F40 motion capture system at University of California [2]

\section{A. on-body radio tracking}

The wireless localization functionality in radiolocation systems typically relies on the analysis of radio signals transmitted with respect to multiple anchors or to other mobile devices (shown in Fig .4). The methods of localization based on radio are usually divided into the following categories: Time Of Arrival (TOA) of the transmitted signal, the Time Difference of Arrival (TDOA), which can be formed out of TOA estimates at synchronized receivers, and the Received Signal Strength Indicator (RSSI), which is based on the distancedependent average power loss.

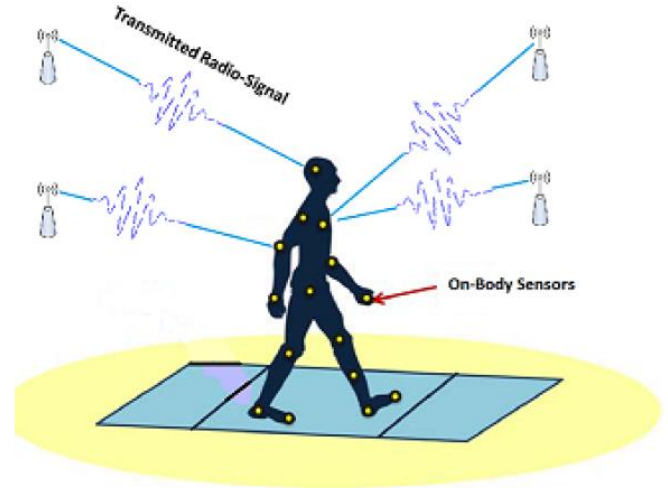

Figure 4. System deployment for on-body radio tracking [4]

\section{B. TOA estimation over IR-UWB}

For positioning systems employing UWB radios, timebased schemes provide very good accuracy due to the high time resolution (large bandwidth) of UWB signals [2]. In TOA localization systems, MT localization is achieved by estimating the radio propagation times related to the MT location. These are obtained by exchanging radio signals with $\mathrm{N}$ fixed base stations or access points (APs), which are placed in known positions. Localization is then obtained by tri-lateration (ie $\mathrm{N}=3$ ) using only the minimum number of APs, or by multi-lateration (ie $\mathrm{N}>3$ ) to increase the method robustness. In the indoor UWB mobile systems, the radio localization based on time is worsened by non-line-of-sight (NLOS) conditions due to signal blocking. In NLOS conditions the first arrivals can be heavily softened, so a strong bias in delay estimation is introduced. The scenario of UWB signal propagation and principle of localization are shown in Fig .5 and Fig .6 respectively. 


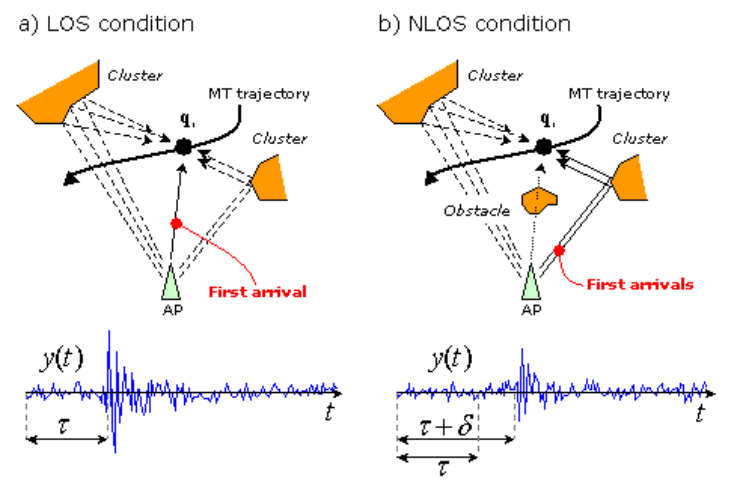

Figure 5. The scenario of UWB signal propagation

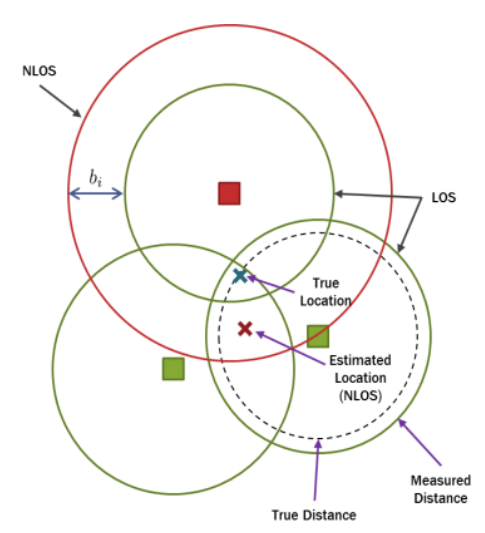

Figure 6. Principal of localization

\section{On-body radio tracking in in WBANs}

The localization based on TOA estimation over IR-UWB links, can directly reflect peer to peer ranges between radio devices. These measurements then subsequently feed positioning or tracking algorithms to deliver the coordinates of mobile nodes in a given reference system. Fig .7 shows an example of typical scenario and system deployment for on-body radio tracking, which could be applied in a WBAN-oriented context. The final positional precision is obviously related to the level of ranging precision over unitary single links. For radio signals propagating at celerity $\mathrm{c}$, the distance between a transmitter and a receiver is straightforwardly given by the product of the Arrival of Time (TOA) and $c$. In an ideal synchronous case, the TOA, so defined as the elapsed time for propagating the radio signal from the transmitter to the receiver, would be simply given by: $T O A_{i}=t_{i}-t_{0}$, where $t_{0}$ is the time instant at which the transmitter starts transmitting and $t_{i}$ is the TOA at the receiver, estimated locally in the observation window and defined according to the local timeline.

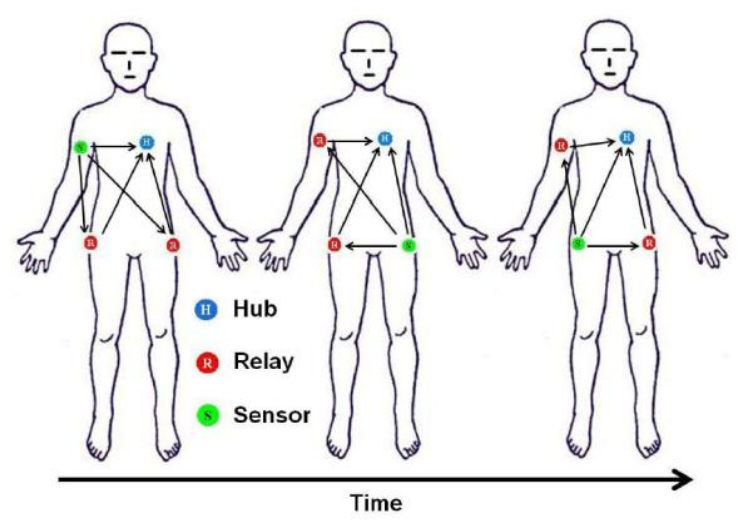

Figure 7. WBANs configuration [13]

Generally, in a WSN, the positions of some sensors are known as anchor nodes, while there are some other sensors as source nodes whose positions are unknown and thus must be estimated using sensor localization. We can estimate the sensor localization via noisy measurements in a WSN. Sensor localization can be divided into two general cases: cooperative and non-cooperative. In the non-cooperative case, source nodes are only connected to anchor nodes and the position of each source node is estimated independently [2]. Generally, the algorithms proposed for cooperative localization are divided into centralized or distributed. In centralized cooperative algorithms, the measurements are sent to a central processor and the location of all source nodes are estimated simultaneously. Whereas in distributed algorithms, each source node is localized independently and estimated data are passed through the neighboring sensors. We are currently working on the Cooperative and non-cooperative localization based on UWB TOA. Fig .8 is a schematic diagram of the location principle based on the topology of wireless sensor networks.

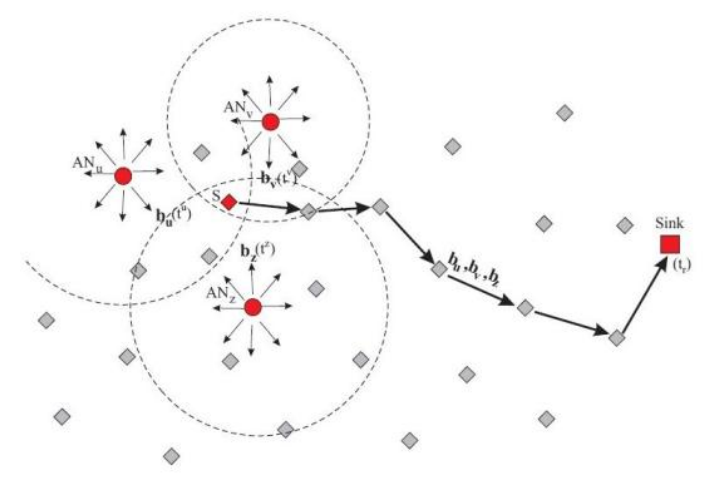

Figure 8. Localization of WSNs [7]

\section{GAIT ANALYSIS BASED ON WBANS}

We attach IMUs to the leg yield an almost arbitrary orientation of the IMU towards its segment, as illustrated in Fig .9. Then, the data calculated from the inertial measurement data are transformed into joint-related 
coordinate systems, in which one or two axes coincide with the joint axis and the longitudinal axis of the segment. The hip joint angle and the knee joint angle can be calculated from the appropriate inertial measurement data. However, this is a challenging job to complete the IMUbased joint angle measurement, specifically on non hingetype joints. Combined with UWB WBANs, the localization of sensor nodes are collected and the kinematic of the lower limb can be exploited. In addition, we can also estimate the torque and power of the movement in each joint of the lower limb based on the information provided by the gyroscopes. Combined with two independent gyroscopes, placed distal and proximally to the joint of interest, the absolute angular velocity of joint in its active axis can be measured.Fig .9 illustrates the placement of the gyroscopes. With the gyroscopes in this position is possible to measure the following movements of the lower limb: Hip flexton, Hip extension, Knee flexton, Knee extension, Ankle dorstflexton, Ankle plantarflexton and so on. (a)

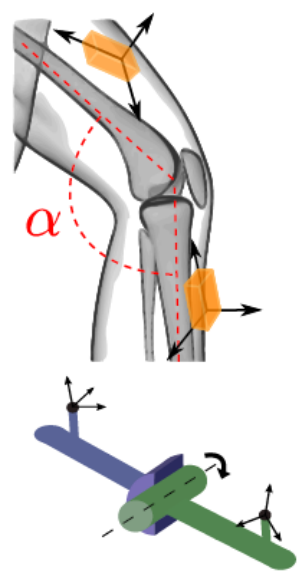

(b)

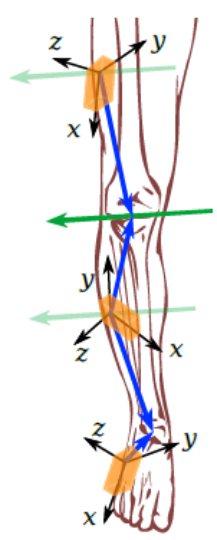

Figure 9. The placement of the gyroscopes [12]

In the lower limb model, six DOFs were considered, three at the hip joint, one at the knee and two for the ankle. We first define the coordinate frames according to the $D-H$ convention. The base frame is positioned at the centre of the pelvis between the hips, at base $X-Y-Z$. The hip frames correspond to the following rotations, as indicated in Fig .10. Three different coordinate systems were used. First, the sensor coordinate system represents the three orthogonal axes in which the acceleration and angular velocity are measured. Second is the segment coordinate system for the body segments that each sensor is attached to and last is the global coordinate system [8].

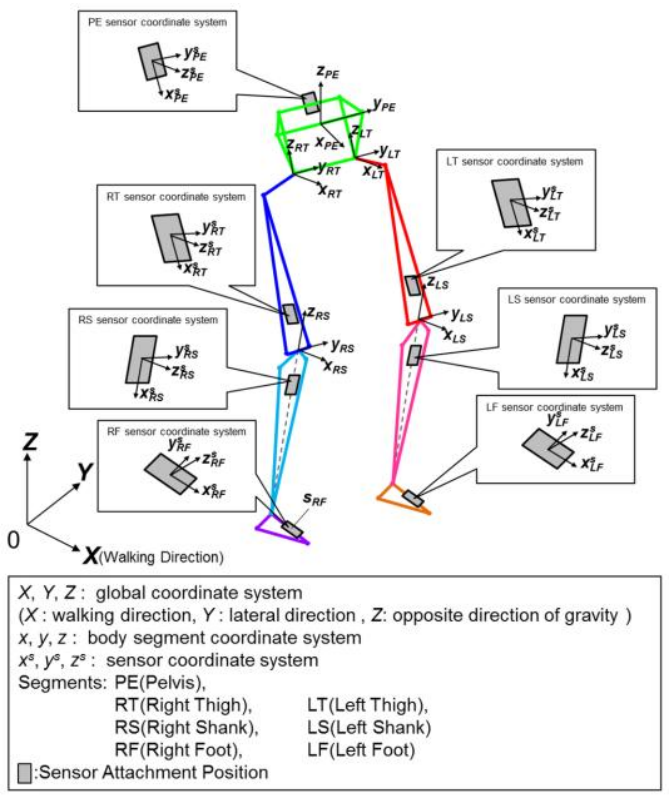

Figure 10. Scheme of the sliding mode control [8]

\section{CONCLUSION}

This paper proposes a method for three dimensional gait analysis using UWB WBANs. The traditional approach based video capture has a low accuracy and a high cost. TOA estimation based on UWB WBANs has high temporal resolution capabilities because of short transmitted pulses whose duration is on the order of a few nanoseconds. Thus, it is best choices for accurate range measurements between on-body devices in the general WBANs context, especially in terms of both the required transmission ranges and relative levels of precision.

\section{ACKNOWLEDGMENT}

This work was financially supported by Chunhui Project from the Ministry of Education of China (Z2012061) and Science Foundation of Department of Education from Jilin Province (No. 2014301).

\section{REFERENCES}

[1] Patwari, N.; Ash, J.N.; Kyperountas, S.; Hero, AO.; Moses, R.L. Correal, N.S., "Locating the nodes: cooperative localization in wireless sensor networks," Signal Processing Magazine, IEEE, vol.22, no.4, pp.54,69, July (2005).

[2] Patwari, N.; Ash, J.N.; Kyperountas, S.; Hero, AO.; Moses, R.L. Correal, N.S., "Locating the nodes: cooperative localization in wireless sensor networks," Signal Processing Magazine, IEEE vol.22, no.4, pp.54,69, July 2005

[3] http://dmnnewswire.digitalmedianet.com/article/UC-MercedAdopts-VICON-F40-Motion-Capture-System-643459

[4] Min Chen, Sergio Gonzalez, Athanasios Vasilakos, Huasong Cao, Victor C. M. Leung, Body Area Networks: A Survey, Mobile Networks and Applications, 16(2), 2011..

[5] Jihad HAMIE, Contributions to Cooperative. Localization Techniques within Mobile. Wireless Body Area Networks, thesis from the University of Nice - Sophia Antipolis, France, 2013.

[6] Monica Nicoli and Vittorio Rampa, Bayesian Algorithms for Indoor Radio Localization, Special: Technology-Enhanced Learning, ERCIM NEWS 71 October 2007.

[7] FIRB - VICom Project, Radio localization of mobile terminals in wireless networks, http://risorse.dei.polimi.it/dsp/tlc/position.htm. 
[8] S. Capkun, S. Ganeriwal, F. Anjum and M. Srivastava, Secure RSS-based Localization in Sensor Networks, Technical Report 529. ETH Zürich, 092006

[9] Shigeru Tadano, Ryo Takeda and Hiroaki Miyagawa, Three Dimensional Gait Analysis Using Wearable Acceleration and Gyro Sensors Based on Quaternion Calculations, Sensors 2013, 13(7), 9321-9343.

[10] Shivam Wadhwa1, Kusum Dangi, Location Based Store and Forward Packet Routing Algorithm for Wireless Body Area Networks: A Survey, International Journal of Computer Engineering and Technology, 5(1),2014.

[11] Kamya Yekeh Yazdandoost, Kamran Sayrafian-Pour, Channel Model for Body Area Network (BAN),

http://www.ece.ubc.ca/_minchen/min paper/Min-0-JNL-2-9-BAN-

\section{MONET2010.pdf}

[12] Seel, T.; Raisch, J.; Schauer, T. IMU-Based Joint Angle Measurement for Gait Analysis. Sensors 2014, 14, 6891-6909.

[13] Dong, Jie and Smith, David. Coexistence and Interference Mitigation for Wireless Body Area Networks: Improvements using On-Body Opportunistic

Relaying . In CoRR, (abs/1305.6992) Year 2013.

[14] Al-Zahrani KS, Bakheit MO, A historical review of gait analysis, Neurosciences (Riyadh). 2008 Apr;13(2):105-8.

[15] Muro-de-la-Herran A, Garcia-Zapirain B, Mendez-Zorrilla A. Gait Analysis Methods: An Overview of Wearable and Non-Wearable Systems, Highlighting Clinical Applications. Sensors. 2014; 14(2):3362-3394. 